\title{
Representativeness of Phase III Trial for Osimertinib in Pretreated Advanced EGFR-Mutated Non-small-cell Lung Cancer Patients and Treatment Outcomes in Clinical Practice
}

\author{
Lishi Lin ${ }^{1}$ (D Egbert F. Smit ${ }^{2,3}$. Adrianus J. de Langen ${ }^{2}$ Dorieke E. M. van Balen ${ }^{1}$ · Jos H. Beijnen ${ }^{1,4}$. \\ Alwin D. R. Huitema ${ }^{1,5,6}$
}

Accepted: 27 November 2021 / Published online: 11 December 2021

(c) The Author(s) 2021

\begin{abstract}
Background Overall survival (OS) data of osimertinib in pretreated non-small-cell lung cancer (NSCLC) in real-world practice is limited, and treatment benefits for patients not represented in the pivotal trials (ineligible) are unclear.

Objective To determine the representativeness of the AURA3 trial for NSCLC patients treated with osimertinib in a realworld setting and to determine outcomes of patients who were represented in the AURA3 trial (eligible) and those who were ineligible.

Methods Advanced NSCLC patients receiving post first-line osimertinib were included in this retrospective study and were divided into two groups based on eligibility criteria of the AURA3 trial. Progression-free survival (PFS) and OS were estimated using the Kaplan-Meier method. Cox models were used to estimate the association of eligibility criteria with OS. Results 328 patients were included; 126 (38\%) patients were eligible and 202 (62\%) patients were ineligible. The most common ineligibility reasons were the number of earlier treatment lines and an Eastern Cooperative Oncology Group performance status (ECOG PS) $>1$. PFS of eligible and ineligible patients was not statistically different (8.0 vs. 5.8 months, $p=0.062)$. Eligible patients had a longer OS ( 24.0 vs. 15.4 months, $p=0.001)$ compared to ineligible patients. ECOG PS was the best predictor for OS. An ECOG PS of 1 was already associated with poorer survival compared to an ECOG PS of 0 (hazard ratio $1.54 ; p=0.016$ ).

Conclusion The majority of the study population was not represented in the AURA3 trial. Survival outcomes of eligible patients are in concordance with the AURA3 trial, while OS of ineligible patients was significantly shorter compared to eligible patients.
\end{abstract}

Lishi Lin

1.lin@nki.nl

1 Department of Pharmacy and Pharmacology, The Netherlands Cancer Institute-Antoni van Leeuwenhoek Hospital, Plesmanlaan 121, 1066 CX Amsterdam, The Netherlands

2 Department of Thoracic Oncology, The Netherlands Cancer Institute-Antoni van Leeuwenhoek Hospital, Amsterdam, The Netherlands

3 Department of Pulmonology, Leiden University Medical Center, Leiden, The Netherlands

4 Department of Pharmaceutical Sciences, Utrecht University, Utrecht, The Netherlands

5 Department of Pharmacology, Princess Máxima Center for Pediatric Oncology, Utrecht, The Netherlands

6 Department of Clinical Pharmacy, University Medical Center Utrecht, Utrecht University, Utrecht, The Netherlands

\section{Key Points}

Real-world outcomes of patients eligible for AURA3 were similar to AURA3 results.

Survival of patients in poor clinical condition was shorter, but still impressive.

Performance status was the best predictor for survival.

\section{Introduction}

Epidermal growth factor receptor tyrosine kinase inhibitors (EGFR TKIs) have been provided to patients with advanced stage non-small-cell lung cancer (NSCLC) with 
activating EGFR mutations for almost two decades now $[1,2]$. The use of EGFR TKIs is, however, accompanied by the acquisition of drug resistance, meaning that all patients will eventually develop disease progression during treatment. The most commonly observed mechanism of resistance to first- and second-generation EGFR TKI treatment is the T790M mutation in EGFR exon 20, which is found in $50-60 \%$ of the NSCLC patients treated with a first- or second-generation EGFR TKI [3].

The third-generation EGFR TKI, osimertinib, was the first to overcome this T790M resistance mutation. It showed a significantly longer progression-free survival (PFS) compared to platinum-pemetrexed chemotherapy as second-line treatment after first-line treatment with a first-generation EGFR TKI in the AURA3 trial (NCT02151981) [4]. In the overall survival (OS) analysis, no statistically significant difference was observed between the study arms. This was attributed to a high crossover rate of patients from the chemotherapy arm to the osimertinib arm, suggesting that patients who progressed on platinum-pemetrexed chemotherapy also benefitted from osimertinib treatment as third-line treatment [5].

In addition to the AURA 3 trial determining the efficacy of second-line osimertinib treatment, it is of relevance to perform observational cohort studies determining the effectiveness in a real-world setting [6]. The study population of randomized clinical trials (RCTs) is generally in a better health state compared to patient populations in clinical practice. This is the result of strict inclusion and exclusion criteria applied in RCTs to improve the valid estimation of treatment effects. The results of RCTs may, therefore, not be representative for patients in clinical practice [7-9]. In addition, treatment benefits are unclear for patients who were not represented in the RCTs [10].

Therefore, our study aimed to determine the representativeness of the AURA3 trial for NSCLC patients treated with osimertinib in routine clinical practice and to determine treatment outcomes of patients who were represented in the AURA3 trial (eligible) and those who were not represented (ineligible).

\section{Materials and Methods}

\subsection{Patients}

This retrospective observational cohort study was conducted at the Netherlands Cancer Institute-Antoni van Leeuwenhoek Hospital (NKI-AvL), Amsterdam. All patients with advanced stage EGFR mutation-positive NSCLC, who received osimertinib as post first-line treatment between July 2015 and
October 2020 were included in this study. Patients who participated in the AURA3 trial were excluded. No other exclusion criteria were applied.

The eligibility criteria of the AURA3 trial were used to assign patients to either the eligible group or the ineligible group [4]. Patients were assigned to the ineligible group if they met one or multiple of the following criteria:

- Negative T790M status confirmed by either a circulating tumor DNA test or a tumor biopsy.

- First-line treatment was not an EGFR TKI or more than one prior treatment line was given.

- Eastern Cooperative Oncology Group performance status (ECOG PS) of $>1$.

- Brain metastases that were unstable, symptomatic, or for which glucocorticosteroids were given within 4 weeks of start of osimertinib treatment.

Data on patient characteristics, tumor characteristics, treatments, and outcomes were extracted from the individual electronic medical records. The data cutoff was 8 March 2021. The conduct of this observational study was approved by the Investigational Review Board of the NKI-AvL and patients' permission to use their data for scientific research was obtained.

\subsection{Outcomes}

The primary endpoints were PFS and OS. PFS was defined as the time from treatment initiation until the first signs of disease progression by either radiology or clinical signs, or death by any cause in the absence of progression. Patients were evaluated for disease status every 3 months and in case of clinical symptoms or worsening of symptoms. OS was defined as the time from treatment initiation until death by any cause. In addition, it was determined whether patients experienced dose-limiting toxicities, which were defined as the need for dose reductions, dose interruptions, or permanent discontinuation of treatment due to adverse events.

\subsection{Statistical Analysis}

Descriptive statistics were reported using frequencies for categorical data and medians for continuous data. Median PFS and OS were calculated using the Kaplan-Meier method, including their 95\% confidence intervals (CIs). Patients who did not have an event of progression or death were censored at the time of the last radiology scan or last follow-up date. A two-sided $p$-value $<0.05$ was considered statistically significant. In addition, OS of patients with brain metastases and leptomeningeal (LM) metastases were determined regardless of eligibility group. The median follow-up time was estimated with the reverse Kaplan-Meier method [11]. 
Univariable and multivariable Cox proportional hazards regression models were used to estimate the association of exclusion criteria and other covariates with OS using the entire study population. The backwards elimination procedure was used starting with all variables of interest to determine the final multivariable model. A two-sided $p$-value $<0.05$ was considered statistically significant. The proportional hazard assumption in the model was investigated using the scaled Schoenfeld residuals. All statistical analyses were performed in $\mathrm{R}$ version 4.0.4 (R Foundation for Statistical Computing, Vienna, Austria).

\section{Results}

\subsection{Characteristics of the Study Population}

In total 328 patients were included in this study. Patient characteristics are depicted in Table 1. Of the patient population, $202(62 \%)$ patients were considered to be ineligible. The majority of ineligible patients met one ineligibility criteria. Thirty-nine (19\%) patients met two ineligibility criteria, whereas 19 (9\%) met more than two of these criteria. The most common causes for ineligibility were the number of earlier treatment lines followed by an ECOG PS $>1$ (Table 2).

\subsection{Treatment Outcomes}

The median duration of follow-up was 27.8 months (range 0-56 months). At the time of data cutoff, 93 patients were still on osimertinib treatment.

Disease progression occurred in $110(87 \%)$ patients in the eligible group and in $176(87 \%)$ patients in the ineligible group. Median PFS of the eligible group was not statistically significantly longer compared to the ineligible group, which was 8.0 months (95\% CI 6.5-10.8) and 5.8 months (95\% CI 4.4-8.0), respectively with a $p$-value of 0.062 (Fig. 1A).

Death was reported for 199 (61\%) patients: 68 (54\%) in the eligible group and $131(65 \%)$ in the ineligible group. Median OS was 24.0 months (95\% CI 21.0-29.5) in eligible patients and 15.4 months (95\% CI 12.5-19.6) in ineligible patients, with a $p$-value of 0.001 (Fig. 1B). Patients with brain metastases had a median OS of 18.2 months (95\% CI 13.5-21.8) and patients with LM metastases had a median OS of 10.1 months (95\% CI 6.5-21.4).

The univariable and multivariable Cox models for the association of exclusion criteria and OS are shown in Table 3. Age, sex, LM metastases, ECOG PS, and EGFR mutation were relevant covariates and were taken into account in the multivariable model. Brain metastasis, T790M status, number of previous treatment lines, and smoking status did not have a significant effect in the
Table 1 Patient characteristics of eligible and ineligible patients

\begin{tabular}{|c|c|c|}
\hline & Eligible $(n=126)$ & Ineligible $(n=202)$ \\
\hline \multicolumn{3}{|l|}{ Age, years } \\
\hline Median (range) & $66(42-87)$ & $63(24-85)$ \\
\hline Female sex [no. (\%)] & $90(72)$ & $150(74)$ \\
\hline \multicolumn{3}{|c|}{ Smoking status [no. (\%)] } \\
\hline Never & $74(59)$ & $123(61)$ \\
\hline Ever & $51(40)$ & $75(37)$ \\
\hline Unknown & $1(1)$ & $4(2)$ \\
\hline \multicolumn{3}{|c|}{ Histologic type [no. (\%)] } \\
\hline Adenocarcinoma & $119(94)$ & $177(88)$ \\
\hline Other & $7(6)$ & $25(12)$ \\
\hline \multicolumn{3}{|c|}{ EGFR mutation [no. (\%)] } \\
\hline Exon 19 deletion & $79(63)$ & $117(58)$ \\
\hline Exon $21 \mathrm{~L} 858 \mathrm{R}$ & $42(33)$ & $59(29)$ \\
\hline Other & $5(4)$ & $22(11)$ \\
\hline Unknown & - & $4(2)$ \\
\hline \multicolumn{3}{|c|}{ T790M mutation [no. (\%)] } \\
\hline Positive & $119(94)$ & $127(63)$ \\
\hline Negative & - & $28(14)$ \\
\hline Unknown & $7(6)$ & $47(23)$ \\
\hline \multicolumn{3}{|l|}{ Metastases [no. (\%)] } \\
\hline Brain & $14(11)$ & $104(52)$ \\
\hline Leptomeningeal & $3(2)$ & $38(19)$ \\
\hline \multicolumn{3}{|c|}{$\begin{array}{l}\text { Prior EGFR TKI use [no. } \\
(\%)]\end{array}$} \\
\hline Erlotinib & $87(69)$ & $132(65)$ \\
\hline Gefitinib & $30(24)$ & $48(24)$ \\
\hline Afatinib & $9(7)$ & $32(16)$ \\
\hline Rociletinib & - & $10(5)$ \\
\hline \multicolumn{3}{|l|}{ ECOG PS [no. (\%)] } \\
\hline 0 & $42(33)$ & $48(24)$ \\
\hline 1 & $82(65)$ & $81(40)$ \\
\hline 2 & - & $51(25)$ \\
\hline 3 or 4 & - & $22(11)$ \\
\hline NA & $2(2)$ & - \\
\hline
\end{tabular}

ECOG PS Eastern Cooperative Oncology Group performance status, $E G F R$ epidermal growth factor receptor, TKI tyrosine kinase inhibitor

multivariable Cox model and were left out. Of the exclusion criteria applied in this study, a higher ECOG PS was correlated strongest with poorer survival, whereas sex, the presence of LM metastases, and the type of EGFR mutation also influenced OS significantly.

\subsection{Toxicity and Adverse Events}

There were no significant differences in the occurrence of dose-limiting toxicities between eligible and ineligible patients. The osimertinib dose was reduced in 35 (10.7\%) 
Table 2 Characteristics on which patients were deemed ineligible

\begin{tabular}{ll}
\hline Earlier treatment & $110(54 \%)$ \\
$1^{\mathrm{a}}$ & $12(6 \%)$ \\
2 & $71(35 \%)$ \\
3 or more & $27(13 \%)$ \\
ECOG PS $>1$ & $73(36 \%)$ \\
Brain metastases & b \\
Negative T790M status & $70(35 \%)$ \\
Total reasons for ineligibility & $28(14 \%)$ \\
\hline
\end{tabular}

ECOG PS Eastern Cooperative Oncology Group performance status

${ }^{a}$ First-line treatment was not with an earlier generation epidermal growth factor receptor tyrosine kinase inhibitor

${ }^{\mathrm{b}}$ Unstable, symptomatic, or requiring corticosteroid use within 4 weeks of start osimertinib treatment

patients due to adverse events. Gastrointestinal toxicity, mainly diarrhea, was the reason for dose reductions in almost half of these cases. Treatment was interrupted in $19(5.8 \%)$ patients, due to diarrhea, skin toxicity, reduced renal function, and pneumonitis. After dose interruption, the treatment was restarted at the initial dose or at a reduced dose. Osimertinib treatment was permanently discontinued in six $(1.8 \%)$ patients. Three of them had an osimertinibinduced pneumonitis. The other reasons for discontinuation were cardiotoxicity, hepatotoxicity, and a combination of gastrointestinal symptoms with other adverse events, which were deemed unbearable by the patient.

\section{Discussion}

This study investigated the clinical outcomes of pretreated EGFR mutation-positive NSCLC patients treated with osimertinib as post first-line treatment in a real-world setting. Approximately $60 \%$ of this study population was deemed ineligible according to the most important AURA3 trial eligibility criteria on which the registration of osimertinib for this indication was based. High ineligibility rates were also seen in other studies, in which the main reasons were poor ECOG PS, unstable or symptomatic brain metastases and specification of previous treatment lines [7-10].

For eligible patients, median OS was similar to the results obtained in the AURA3 trial for the non-Asian population (median OS 20.3; 95\% CI 18.3-26.8) [5]. In addition to this trial, the results for patients with LM metastases closely resembled the results of the BLOOM trial (median OS 11.0; 95\% CI 8.0-18.0) in which the efficacy of double dosage osimertinib was assessed in patients with LM metastases [12]. In another trial assessing the efficacy of double dosage osimertinib in patients with brain and LM metastases, the results for patients with brain metastases (median OS 16.9; 95\% CI 7.9-NA) were similar to our results, whereas results of patients with LM metastases (median OS 13.3; 95\% CI 9.1-NA) were slightly more favorable than ours [13]. However, it has to be noted that caution is still warranted in the comparison with the latter two trials due to differences in study populations, as both trials were performed in an Asian population with ECOG PS $\leq 2$.

Treatment with osimertinib also showed clinical benefit for ineligible patients when comparing survival outcomes to literature. In a cohort study of Polish NSCLC patients, clinical outcomes of first- and second-generation EGFR TKIs as first-line treatment were studied. Median PFS and OS were 11.9 and 19.4 months, respectively, from the time of EGFR TKI treatment initiation, implying a duration of 7.5 months from the initiation of second-line treatment or best supportive care until death [14]. In a Swedish cohort study with NSCLC patient who were treated with first- and second-generation EGFR TKI treatment, the median OS was 15.5 months from the time of diagnosis. The majority of this cohort received a first- or second-generation EGFR TKI as first-line treatment, whereas patients who were diagnosed in the early years of the cohort study may have received chemotherapy as first-line treatment followed by an EGFR TKI as second-line treatment [15]. Taking into consideration that the ineligible patients in our study are the patients in clinical practice with the poorest health and that some of these ineligible patients received osimertinib as thirdline treatment or further lines of treatment, the median OS observed for ineligible patients in our study is impressive.

In the multivariable Cox model, a higher ECOG PS was strongly correlated with a shorter survival in which the hazard ratios roughly doubled per change in ECOG PS. This same trend was seen in a prognostic model based on ECOG PS alone of Jang et al. [16]. Another noteworthy covariate is the difference in sex. Although women generally have better survival outcomes in NSCLC than men, the difference in this study seemed to be larger as compared to other studies $[17,18]$.

With regard to dose-limiting toxicities, it appears that dose reductions occur more often in clinical practice compared to the AURA3 trial, in which dose interruptions were more common compared to dose reductions. In the AURA3 trial, dose interruptions were applied first when patients experienced grade 3 or higher toxicities and/or unacceptable toxicity of any grade, after which osimertinib was restarted at the initial or a reduced dose. After additional analyses of the AURA and AURA2 studies, it became clear that osimertinib did not show an exposure-efficacy relationship at the approved dosing regimen, while it does show an exposuretoxicity relationship [19]. Therefore, dose reductions in clinical practice were applied more often to reduce toxicity, while the effectiveness of osimertinib was preserved. 
Fig. 1 Kaplan-Meier curves for a progression-free survival (PFS) and $\mathbf{b}$ overall survival (OS) according to eligibility. $\mathrm{CI}$ confidence interval

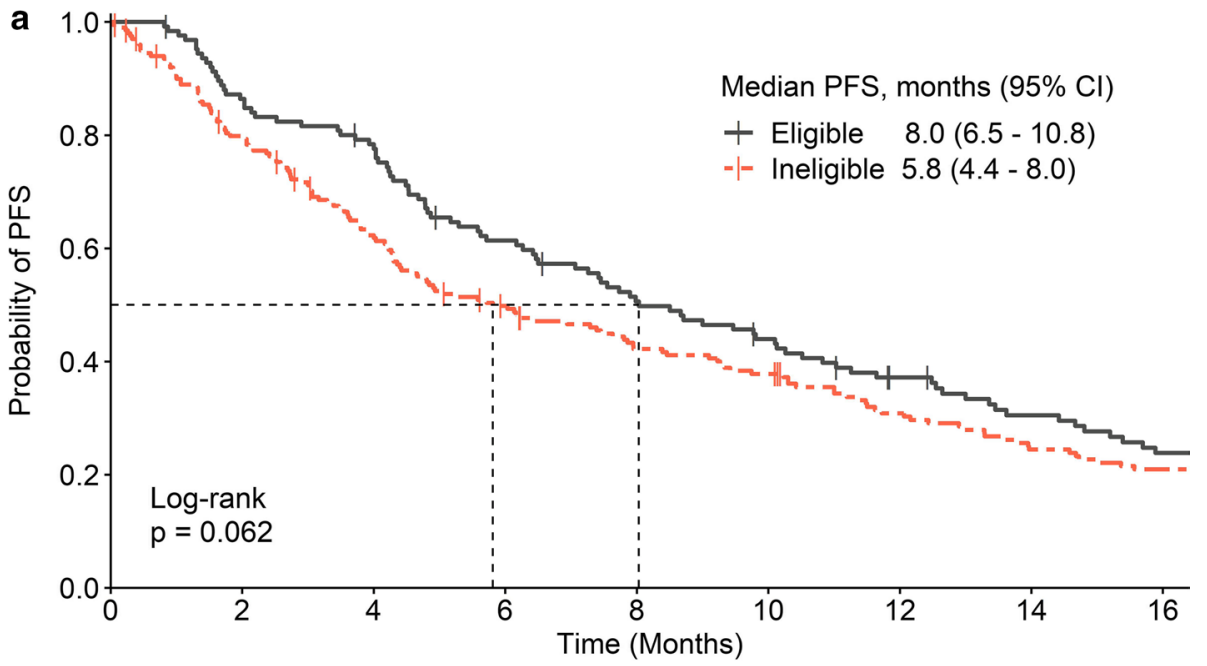

Number at risk

$\begin{array}{rrrrrrrrr}-126 & 108 & 97 & 75 & 61 & 52 & 40 & 32 & 25 \\ -202 & 157 & 120 & 93 & 77 & 69 & 53 & 42 & 36\end{array}$

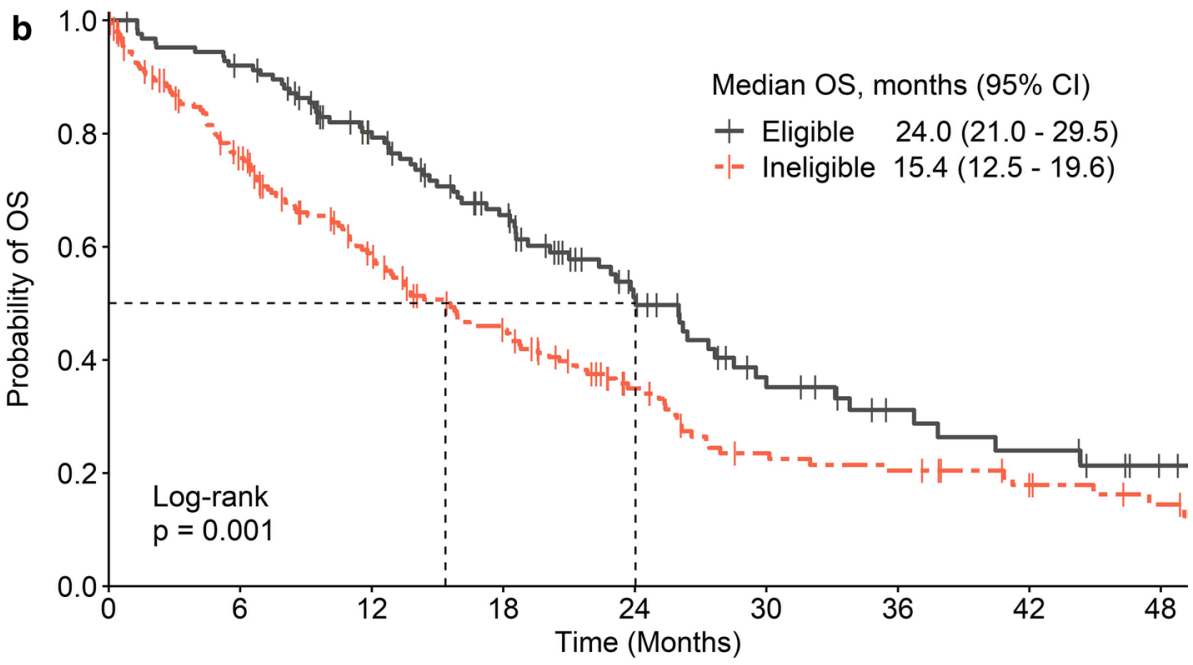

Number at risk

$\begin{array}{rrrrrrrrr}-126 & 114 & 86 & 63 & 37 & 21 & 13 & 10 & 4 \\ -202 & 140 & 95 & 67 & 39 & 23 & 20 & 14 & 8\end{array}$

The number of included patients is relatively large, which is a strength of this study. Despite the large study population, data from other centers are needed to determine whether data are in accordance with ours and to study the differences between ethnicities in a real-world setting [5]. The most important limitation of this study is its retrospective nature, complicating the application of all eligibility criteria of the AURA3 trial. In the current study, patients were deemed ineligible only if this was apparent from the available data. Due to this conservative approach, it is possible that even a larger proportion of the patient population was actually ineligible, and that the benefits of osimertinib treatment in both groups is underestimated in this study.

As there seems to be a large gap between patient populations in RCTs and the patient population in clinical practice, further real-world studies are needed to determine the effectiveness of treatments in ineligible patients to support decision making in clinical practice. This can be quite challenging since oncology is a fast-developing field and the standard of care changes accordingly. Studies comparing the previously standard-of-care treatment with the newly introduced treatment are prone to bias as other changes in the standard of care may act as confounders, which cannot be accounted for to the full extent [6]. In the 
Table 3 Univariable and multivariable Cox model of the association of exclusion criteria and covariates on overall survival

\begin{tabular}{|c|c|c|c|c|c|c|}
\hline & \multicolumn{3}{|c|}{ Univariable } & \multicolumn{3}{|c|}{ Multivariable } \\
\hline & $n$ & $\mathrm{HR}(95 \% \mathrm{CI})$ & $p$-value & $n$ & HR $(95 \% \mathrm{CI})$ & $p$-value \\
\hline \multicolumn{7}{|l|}{ CNS } \\
\hline Absent & 198 & 1 & & & & \\
\hline Asymptomatic & 74 & $1.13(0.80-1.60)$ & 0.473 & & & \\
\hline Symptomatic & 56 & $2.07(1.41-3.03)$ & $<0.001$ & & & \\
\hline \multicolumn{7}{|l|}{ LM } \\
\hline Absent & 287 & 1 & & 282 & 1 & \\
\hline Present & 41 & $2.04(1.36-3.04)$ & 0.001 & 40 & $2.23(1.42-3.49)$ & $<0.001$ \\
\hline \multicolumn{7}{|l|}{ ECOG PS } \\
\hline 0 & 90 & 1 & & 88 & 1 & \\
\hline 1 & 163 & $1.52(1.07-2.14)$ & 0.018 & 161 & $1.54(1.08-2.19)$ & 0.016 \\
\hline 2 & 51 & $2.84(1.86-4.35)$ & $<0.001$ & 51 & $3.02(1.96-4.65)$ & $<0.001$ \\
\hline 3 or 4 & 22 & $6.49(3.58-11.8)$ & $<0.001$ & 22 & $6.50(3.46-12.2)$ & $<0.001$ \\
\hline \multicolumn{7}{|l|}{ T790M status } \\
\hline Positive & 246 & 1 & & & & \\
\hline Negative & 28 & $1.65(0.99-2.73)$ & 0.053 & & & \\
\hline Unknown & 54 & $1.83(1.26-2.67)$ & 0.002 & & & \\
\hline \multicolumn{7}{|l|}{ Treatment lines } \\
\hline 1 & 230 & 1 & & & & \\
\hline 2 & 71 & $0.88(0.63-1.24)$ & 0.469 & & & \\
\hline 3 or more & 27 & $1.48(0.94-2.33)$ & 0.090 & & & \\
\hline Age (years) & 328 & $1.01(1.00-1.02)$ & 0.104 & 322 & $1.02(1.01-1.03)$ & 0.003 \\
\hline \multicolumn{7}{|l|}{ Sex } \\
\hline Male & 88 & 1 & & 85 & 1 & \\
\hline Female & 240 & $0.59(0.44-0.80)$ & 0.001 & 237 & $0.54(0.39-0.74)$ & $<0.001$ \\
\hline \multicolumn{7}{|l|}{ EGFR mutation } \\
\hline Exon 19 del & 196 & 1 & & 194 & 1 & \\
\hline Exon $21 \mathrm{~L} 858 \mathrm{R}$ & 101 & $1.38(1.02-1.88)$ & 0.038 & 101 & $1.52(1.12-2.08)$ & 0.008 \\
\hline Other & 27 & $3.14(2.01-4.90)$ & $<0.001$ & 27 & $3.46(2.17-5.52)$ & $<0.001$ \\
\hline
\end{tabular}

$C l$ confidence interval, $C N S$ central nervous system, ECOG PS Eastern Cooperative Oncology Group performance status, EGFR epidermal growth factor receptor, $H R$ hazard ratio, $L M$ leptomeningeal

case of osimertinib, it would also be of interest to determine the effectiveness of first-line treatment in patients who were not represented in the respective Phase III trial, as treatment outcomes are potentially even better than the results of the ineligible patients in our study.

In addition, future research investigating the use of observational data as synthetic control arms in RCTs would be of interest, especially since only a limited set of exclusion criteria was applied in our study compared to the AURA3 trial and the results of eligible patients were similar to the AURA3 trial. The main advantage of these synthetic controls is the need for a smaller study population in RCTs, in which more patients can be allocated to the intervention arm. A small proportion of patients can then be allocated to the control arm, which will be combined with observational data from clinical practice [20].

In conclusion, the majority of patients with EGFR mutationpositive NSCLC who received osimertinib as post first-line treatment at our institution were not represented in the AURA3 trial. The treatment outcomes of eligible patients are in accordance with the results of the AURA3 trial, while OS of ineligible patients was significantly shorter as compared to eligible patients. However, considering that these patients are in poorer health, the survival outcomes of ineligible patients are impressive.

\section{Declarations}

Funding No external funding was used in the preparation of this article.

Conflict of interest L. Lin, D. E. M. van Balen, and A. D. R. Huitema declare that they have no known competing financial interest or personal relationships that could have influenced the work reported in this paper. E. F. Smit reports a consulting or advisory role (institution) for AstraZeneca, Bayer, Boehringer Ingelheim, Bristol-Myers Squibb, Daiichi Sankyo, Eli Lilly, Merck KGaA, MSD Oncology, Novartis, Roche/Genentech, Seattle 
Genetics, and Takeda; and research funding (institution) from AstraZeneca, Bayer, Boehringer Ingelheim, Bristol-Myers Squibb, and Roche/Genentech. A. J. de Langen reports grants from BMS, grants from MSD, grants from Boehringer, non-financial support from Merck Serono, grants from AstraZeneca, and non-financial support from Roche, outside the submitted work. J. H. Beijnen is a part-time employee and (indirect) shareholder of Modra Pharmaceuticals BV. He is (a part) patent holder of oral taxane formulations that are clinically developed by Modra Pharmaceuticals BV, a spin-off company of the Netherlands Cancer Institute, Amsterdam, the Netherlands. This is not related to this article.

Ethics approval The conduct of this observational study was approved by the Investigational Review Board of the Netherlands Cancer Institute-Antoni van Leeuwenhoek Hospital.

Consent to participate The need for written consent for patients was waived per the above approval protocol.

Consent for publication Not applicable.

Availability of data and material Not applicable.

Code availability Not applicable.

Authors' contributions All authors contributed to the study conception and design. Data collection and analysis were performed by L. Lin, A. D. R. Huitema, E. F. Smit, and A. J. De Langen. The first draft of the manuscript was written by L. Lin and all authors commented on previous versions of the manuscript. All authors read and approved the final manuscript.

Open Access This article is licensed under a Creative Commons Attribution-NonCommercial 4.0 International License, which permits any non-commercial use, sharing, adaptation, distribution and reproduction in any medium or format, as long as you give appropriate credit to the original author(s) and the source, provide a link to the Creative Commons licence, and indicate if changes were made. The images or other third party material in this article are included in the article's Creative Commons licence, unless indicated otherwise in a credit line to the material. If material is not included in the article's Creative Commons licence and your intended use is not permitted by statutory regulation or exceeds the permitted use, you will need to obtain permission directly from the copyright holder. To view a copy of this licence, visit http://creativecommons.org/licenses/by-nc/4.0/.

\section{References}

1. Shepherd FA, Pereira JR, Ciuleanu T, Eng HT, Hirsh V, Thongprasert $\mathrm{S}$, et al. Erlotinib in previously treated non-small-cell lung cancer. N Engl J Med. 2005;353(2):123-32.

2. Thatcher N, Chang A, Parikh P, Pereira JR, Ciuleanu T, Von Pawel J, et al. Gefitinib plus best supportive care in previously treated patients with refractory advanced non-small-cell lung cancer: results from a randomised, placebo-controlled, multicentre study (Iressa Survival Evaluation in Lung Cancer). Lancet. 2005;366(9496):1527-37.

3. Camidge DR, Pao W, Sequist LV. Acquired resistance to TKIs in solid tumours: learning from lung cancer. Nat Rev Clin Oncol. 2014;11(8):473-81.

4. Mok TS, Wu YL, Ahn MJ, Garassino MC, Kim HR, Ramalingam SS, et al. Osimertinib or platinum-pemetrexed in EGFR T790M-positive lung cancer. N Engl J Med. 2017;376(7):629-40.
5. Papadimitrakopoulou VA, Mok TS, Han J-Y, Ahn M-J, Delmonte A, Ramalingam SS, et al. Osimertinib versus platinum-pemetrexed for patients with EGFR T790M advanced NSCLC and progression on a prior EGFR-tyrosine kinase inhibitor: AURA3 overall survival analysis. Ann Oncol. 2020;31:1536-44.

6. Schneeweiss S, Avorn J. A review of uses of health care utilization databases for epidemiologic research on therapeutics. J Clin Epidemiol. 2005;58(4):323-37.

7. Yoo SH, Keam B, Kim M, Kim TM, Kim DW, Heo DS. Generalization and representativeness of phase III immune checkpoint blockade trials in non-small cell lung cancer. Thorac Cancer. 2018;9(6):736-44.

8. Al-Baimani K, Jonker H, Zhang T, Goss GD, Laurie SA, Nicholas G, et al. Are clinical trial eligibility criteria an accurate reflection of a realworld population of advanced non-small-cell lung cancer patients? Curr Oncol. 2018;25(4):e291-7.

9. Kennedy-Martin T, Curtis S, Faries D, Robinson S, Johnston J. A literature review on the representativeness of randomized controlled trial samples and implications for the external validity of trial results. Trials. 2015;16(1):1-14.

10. Kawachi H, Fujimoto D, Morimoto T, Ito M, Teraoka S, Sato Y, et al. Clinical characteristics and prognosis of patients with advanced nonsmall-cell lung cancer who are ineligible for clinical trials. Clin Lung Cancer. 2018;19(5):e721-34.

11. Schemper M, Smith TL. A note on quantifying follow-up in studies of failure time. Control Clin Trials. 1996;17(4):343-6.

12. Yang JCH, Kim SW, Kim DW, Lee JS, Cho BC, Ahn JS, et al. Osimertinib in patients with epidermal growth factor receptor mutationpositive non-small-cell lung cancer and leptomeningeal metastases: the BLOOM study. J Clin Oncol. 2020;38(6):538-47.

13. Park S, Lee MH, Seong M, Kim ST, Kang JH, Cho BC, et al. A phase II, multicenter, two cohort study of $160 \mathrm{mg}$ osimertinib in EGFR T790M-positive non-small-cell lung cancer patients with brain metastases or leptomeningeal disease who progressed on prior EGFR TKI therapy. Ann Oncol. 2020;31(10):1397-404.

14. Pluzanski A, Krzakowski M, Kowalski D, Dziadziuszko R. Realworld clinical outcomes of first-generation and second-generation epidermal growth factor receptor tyrosine kinase inhibitors in a large cohort of European non-small-cell lung cancer patients. ESMO Open. 2020;5(6):1-8.

15. Bergqvist M, Christensen HN, Wiklund F, Bergström S. Real world utilization of EGFR TKIs and prognostic factors for survival in NSCLC during 2010-2016 in Sweden: a nationwide observational study. Int J Cancer. 2020;146(9):2510-7.

16. Jang RW, Caraiscos VB, Swami N, Banerjee S, Mak E, Kaya E, et al. Simple prognostic model for patients with advanced cancer based on performance status. J Oncol Pract. 2014;10(5):e335-41.

17. Pinto JA, Vallejos CS, Raez LE, Mas LA, Ruiz R, Torres-Roman JS, et al. Gender and outcomes in non-small cell lung cancer: an old prognostic variable comes back for targeted therapy and immunotherapy? ESMO Open. 2018;3(3):1-10.

18. Sagerup CMT, Småstuen M, Johannesen TB, Helland Å, Brustugun OT. Sex-specific trends in lung cancer incidence and survival: a population study of 40118 cases. Thorax. 2011;66(4):301-7.

19. Brown K, Comisar C, Witjes H, Maringwa J, de Greef R, Vishwanathan $\mathrm{K}$, et al. Population pharmacokinetics and exposureresponse of osimertinib in patients with non-small cell lung cancer. Br J Clin Pharmacol. 2017;83(6):1216-26.

20. Thorlund K, Dron L, Park JJH, Mills EJ. Synthetic and external controls in clinical trials - a primer for researchers. Clin Epidemiol. 2020;12:457-67. 